\title{
Diabetes Mellitus and Its Effects on All-Cause Mortality After Radiopeptide Therapy for Neuroendocrine Tumors
}

\author{
Maria Umlauft*1,2, Piotr Radojewski*1, Petar-Marko Spanjol ${ }^{1}$, Rebecca Dumont ${ }^{1}$, Nicolas Marincek ${ }^{1,3}$, Attila Kollar ${ }^{4}$, \\ Philippe Brunner ${ }^{3}$, Jan Beyersmann ${ }^{2}$, Jan Müller-Brand ${ }^{3}$, Helmut R. Maecke ${ }^{5}$, Markus Laimer ${ }^{6}$, and Martin A. Walter ${ }^{1,3}$ \\ ${ }^{1}$ Institute of Nuclear Medicine, University Hospital Bern, Bern, Switzerland; ${ }^{2}$ Institute of Statistics, Ulm University, Ulm, Germany; \\ ${ }^{3}$ Institute of Nuclear Medicine, University Hospital Basel, Basel, Switzerland; ${ }^{4}$ Division of Medical Oncology, University Hospital \\ Bern, Bern, Switzerland; ${ }^{5}$ Department of Nuclear Medicine, University Hospital Freiburg, Freiburg, Germany; and ${ }^{6}$ Division of \\ Endocrinology, Diabetes \& Clinical Nutrition, University Hospital Bern, Bern, Switzerland
}

\begin{abstract}
We aimed to assess the risk of developing diabetes mellitus and its effects on all-cause mortality after radiopeptide therapy for neuroendocrine tumors (NETs). Methods: NET patients received somatostatin radiopeptide therapy with ${ }^{90}$ Y-DOTATOC or ${ }^{177}$ Lu-DOTATOC. The incidence of diabetes mellitus and its mortality were assessed using univariate and multivariate regression. Results: Overall, 1,535 NET patients were enrolled and received 3,807 treatment cycles. After treatment, 72 patients developed diabetes mellitus, including 47 cases after ${ }^{90}$ Y-DOTATOC and 25 cases after combined treatment. The diabetes mellitus risk was higher before than after DOTATOC (estimate, 0.0032; $P<0.001$ ), and overall survival was similar in patients with and without diabetes mellitus (hazard ratio, 1.13; 95\% confidence interval, 0.91-1.39; $n=1,535 ; P=0.27$ ). Conclusion: Radiopeptide therapy does not appear to increase the risk of developing diabetes mellitus in NET patients, whereas diabetes mellitus does not appear to increase the mortality of NET patients undergoing receptor-targeted radiopeptide therapy.
\end{abstract}

Key Words: sstr $_{2}$; metabolic therapy; targeted therapy; adverse event; toxicity

J Nucl Med 2017; 58:97-102

DOI: 10.2967/jnumed.116.180687

\section{$\mathbf{R}$} adiolabeled somatostatin analogs are successful examples of targeted tumor therapy (Fig. 1) (1), which can achieve meaningful clinical responses in patients with locally advanced and metastatic neuroendocrine tumors (NETs) (2-4).

NET patients are generally at risk of experiencing impaired glucose metabolism from a variety of first-line therapies. Pancreatic surgery reduces the amount of functional parenchyma, streptozotocin is cytotoxic to pancreatic $\beta$-cells, octreotide and everolimus reduce insulin secretion, and everolimus also increases insulin resistance $(5,6)$. A potential consequence of these therapies is the onset of prediabetes or diabetes mellitus, which is commonly associated with increased long-term, all-cause mortality in oncologic patients (7).

Received Jul. 5, 2016; revision accepted Aug. 22, 2016.

For correspondence or reprints contact: Martin A. Walter, Institute of Nuclear Medicine, University Hospital Bern, CH-3010 Bern, Switzerland.

E-mail: m.a.walter@gmx.net

${ }^{*}$ Contributed equally to this work.

Published online Sep. 15, 2016.

COPYRIGHT (C 2017 by the Society of Nuclear Medicine and Molecular Imaging.
The high expression of somatostatin receptors by the pancreas, and especially in the pancreatic islets $(8)$, has led to the theory that treatment with radiolabeled somatostatin analogs might cause destruction of endocrine pancreatic parenchyma and subsequent development of diabetes mellitus. However, the incidence of diabetes after radiopeptide therapy and its effect on all-cause mortality in NET patients remained unknown.

Herein, we report the risk of developing diabetes mellitus after treatment with radiolabeled somatostatin analogs and its effects on all-cause mortality, to provide a framework for improved clinical management of NET patients.

\section{MATERIALS AND METHODS}

\section{Patients}

We performed a cross-sectional post hoc follow-up on diabetesrelated outcomes in NET patients who had been enrolled in a prospective trial investigating the benefit of treatment with radiolabeled DOTATOC (tetraazacyclododecane-tetraacetic acid [DOTA]-modified somatostatin analog $\mathrm{Tyr}^{3}$-octreotide [TOC]), performed between 1997 and 2010.

Patients had been enrolled and treated as previously described (2-4). In brief, patients with progressive metastatic NET and tumor uptake in somatostatin receptor imaging were enrolled to receive somatostatin receptor-targeted radiopeptide therapy with ${ }^{90}$ Y-DOTATOC, ${ }^{177} \mathrm{Lu}$-DOTATOC, or their combination at the University Hospital Basel, Switzerland. Patients were excluded in the case of concurrent antitumor treatment, pregnancy, breast-feeding, urinary incontinence, preexisting grade $3 / 4$ hematologic toxicities, and severe concomitant illness.

The study was designed and performed according to good clinical practice, Swiss drug laws, and the Declaration of Helsinki. It was approved by the Basel ethics committee for human studies (\#120/ 1997) and registered (ClinicalTrials.gov number NCT00978211). Written informed consent was obtained from all participants or legal representatives.

\section{Follow-up and Outcomes}

Primary outcome was the diagnosis of diabetes mellitus, before or after the start of DOTATOC treatment, made by the referring centers or primary practitioners. Data on the diagnosis of diabetes mellitus were actively obtained from the referring physicians and primary practitioners and approved for completeness at the study center.

Secondary outcome was survival after DOTATOC in patients with and without diabetes mellitus. Survival data were obtained from referring physicians, primary practitioners, or the patient and approved for completeness at the study center. 


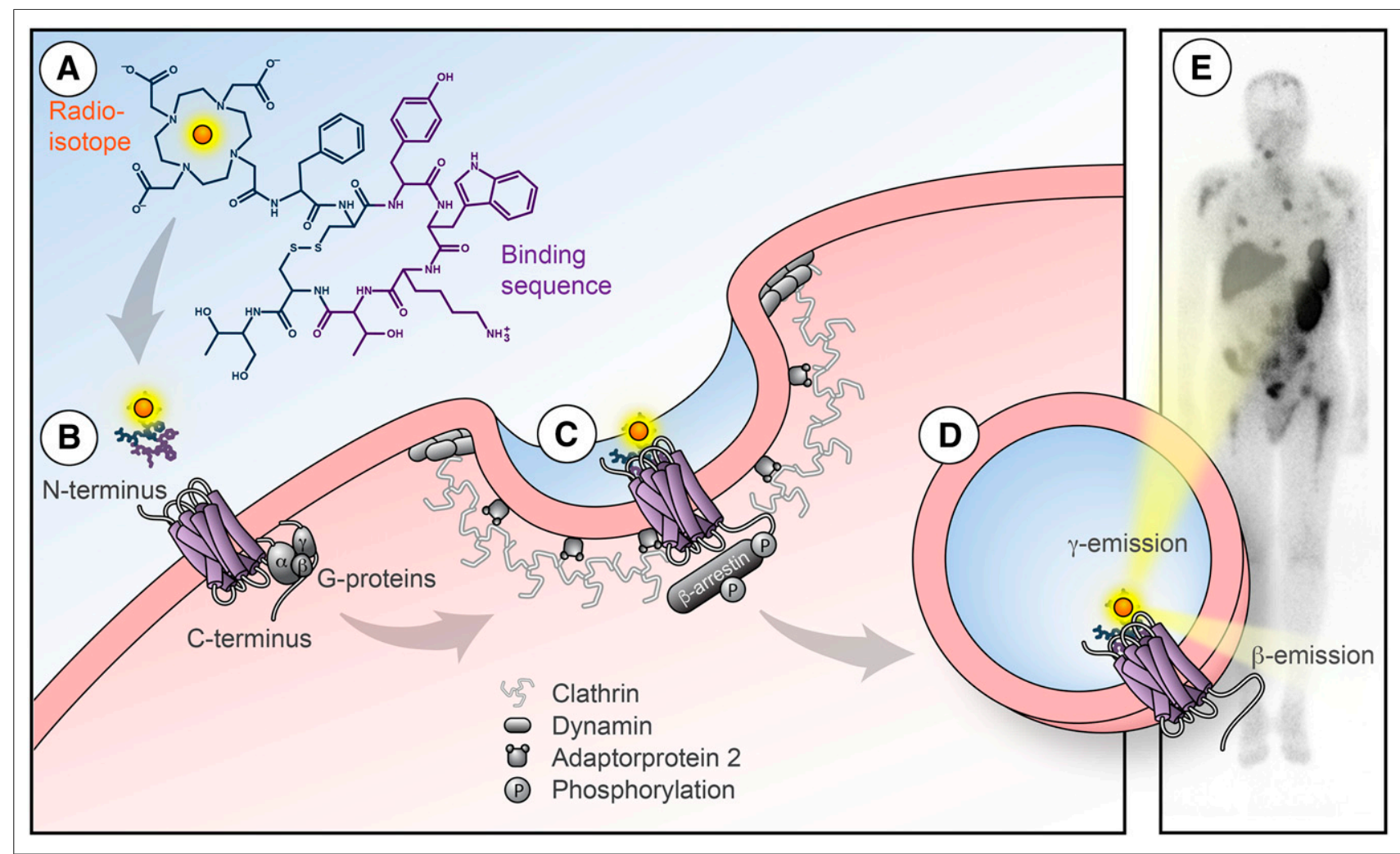

FIGURE 1. (A) DOTATOC comprises somatostatin analog TOC and chelator DOTA for radiolabeling. Radiolabeled DOTATOC is injected intravenously and binds to G-protein-coupled somatostatin receptor subtype 2 on cell surface (B), internalizes via clathrin-mediated endocytosis (C), and enables tumor treatment via $\beta^{-}$-emission (D). (E) Detection of $y$-emission allows visualization of tumor uptake and biodistribution of radiolabeled DOTATOC. Potential adverse events may result from biodistribution of DOTATOC.

Additional outcomes were changes in the serum levels of glucose and C-peptide after the start of DOTATOC treatment. They were investigated in all patients receiving ${ }^{177} \mathrm{Lu}$-DOTATOC and in age- and sex-matched patients who received ${ }^{90} \mathrm{Y}$-DOTATOC.

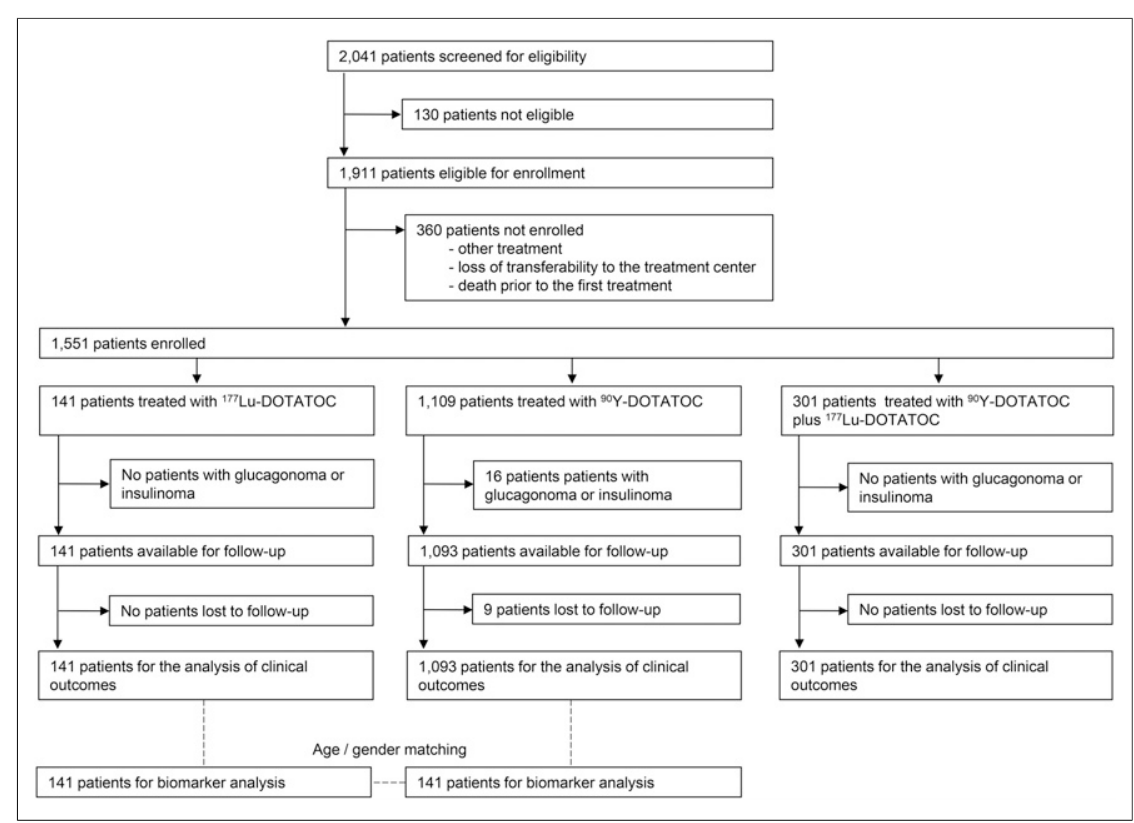

FIGURE 2. Study profile.

\section{Blood Samples}

Blood samples were drawn before each treatment cycle from the nonfasting patient and immediately centrifuged. Blood serum was collected and stored at $-20^{\circ} \mathrm{C}$. Serum glucose (Glucose GODPAP; Roche Diagnostics) and C-peptide (IMMULITE 2000 Immunoassay System; Siemens Healthcare) were measured in duplicate with standardized protocols using 1 assay batch.

\section{Statistical Analysis}

The primary hypothesis was an increased incidence of diabetes mellitus after DOTATOC. The incidence before and after DOTATOC was compared via estimation of difference between a binomial- and the Aalen-Johansen estimator. The incidence before DOTATOC was calculated conditional on study entry. To include groups without diabetes cases, proportional hazard regression was used as previously described, and profile likelihood-based confidence intervals were calculated for confirmation $(9,10)$. Covariables were sex (female vs. male), age (per y), histology (pancreatic NET vs. carcinoids vs. rare NET), disease duration (in mo), pretreatment (surgery vs. chemotherapy vs. radiotherapy), treatment regimen ${ }^{90} \mathrm{Y}$-DOTATOC vs. ${ }^{177} \mathrm{Lu}$-DOTATOC vs. their combination), and 
cumulative administered activity (per GBq). Insulinoma and glucagonoma cases were excluded because of their abnormal glucose metabolism. Subgroup analyses were performed to compare the incidence of diabetes mellitus after ${ }^{90}$ Y-DOTATOC, ${ }^{177} \mathrm{Lu}$-DOTATOC, or their combination. Intention-to-treat analysis was applied. A worst-case-scenario analysis was performed assuming diabetes mellitus developed in all patients who were lost to follow-up.

The secondary hypothesis was a shorter survival in NET patients with diabetes mellitus. The survival with and without diabetes mellitus was compared using multivariate Cox regression with the covariables listed above. Sensitivity analyses in patients with and without pancreas surgery were performed for all analyses to detect effects of pancreas surgery.

Glucose and C-peptide serum profiles were generated via the random intercept model and smoothed using the local regression method (11). Two-sided $P$ values below 5\% were considered statistically significant. Effect estimates were expressed as hazard ratios (HRs) with $95 \%$ confidence intervals (CIs).

\section{RESULTS}

Between February 1997 and February 2010, 2,041 patients were screened for eligibility. Of these, 130 patients $(6.4 \%)$ were not eligible because of absent visible tumor uptake in pretherapeutic imaging, poor physical condition, or impaired bone marrow function. Furthermore, 360 patients $(17.6 \%)$ were eligible but not enrolled, because they received other treatment, lost transferability to the treatment center, or died before the first treatment cycle (Fig. 2). The remaining 1,551 patients (76.0\%) were enrolled from more than 100 centers in 29 countries and were treated with 3,807 treatment cycles (median, 2 cycles per patient; range, $1-11$ cycles per patient). Eight insulinoma patients $(0.4 \%)$ and 8 glucagonoma patients $(0.4 \%)$ were excluded from the analyses. The characteristics of the included 1,535 patients are shown in Table 1.

\section{Risk of Developing Diabetes Mellitus}

At the time of enrollment, 186 of 1,535 patients (12.1\%) had a preexisting diabetes mellitus. In the remaining 1,349

TABLE 1

Patient Baseline Characteristics $(n=1,535)$

\begin{tabular}{|c|c|c|c|c|}
\hline Characteristic & $\begin{array}{c}{ }^{90} \text { Y-DOTATOC } \\
(n=1,093)\end{array}$ & $\begin{array}{c}{ }^{177} \text { Lu-DOTATOC } \\
(n=141)\end{array}$ & $\begin{array}{c}\text { 177Lu-DOTATOC } \\
\text { plus }{ }^{90} \text { Y-DOTATOC } \\
(n=301)\end{array}$ & $\begin{array}{l}\text { All patients } \\
(n=1,535)\end{array}$ \\
\hline \multicolumn{5}{|l|}{ Sex } \\
\hline Female & $471(43.1 \%)$ & 59 (41.8\%) & $120(39.9 \%)$ & $650(42.3 \%)$ \\
\hline Male & $622(56.9 \%)$ & 82 (58.2\%) & $181(60.1 \%)$ & 885 (57.7\%) \\
\hline \multicolumn{5}{|l|}{ Age $(y)$} \\
\hline Median & 58.9 & 62.4 & 59.0 & 59.2 \\
\hline Range & $11.2-86.5$ & $14.8-83.4$ & $23.2-82.1$ & $11.2-86.5$ \\
\hline \multicolumn{5}{|l|}{ Disease duration (mo) } \\
\hline Median & 22.8 & 16.6 & 18.7 & 21.8 \\
\hline Range & $0.0-460.0$ & $1.2-374.7$ & $0.8-623.4$ & $0.0-623.4$ \\
\hline \multicolumn{5}{|l|}{ Pretreatment } \\
\hline Surgery & 597 (54.6\%) & 79 (56.0\%) & $194(64.5 \%)$ & $870(56.7 \%)$ \\
\hline Chemotherapy & $344(31.5 \%)$ & 46 (32.6\%) & $82(27.2 \%)$ & $472(30.7 \%)$ \\
\hline Radiation & $143(13.1 \%)$ & 17 (12.1\%) & $31(10.3 \%)$ & 191 (12.4\%) \\
\hline Pancreas surgery & $301(27.5 \%)$ & 25 (17.7\%) & $81(26.9 \%)$ & 407 (26.5\%) \\
\hline \multicolumn{5}{|l|}{ DOTATOC cycles } \\
\hline Median & 2 & 2 & 3 & 2 \\
\hline Range & $1-10$ & $1-5$ & $2-11$ & $1-11$ \\
\hline \multicolumn{5}{|c|}{ Cumulative activity in $\mathrm{MBq}(\mathrm{mCi})$} \\
\hline Median & $12,950(350)$ & $14,800(400)$ & $22,200(600)$ & $14,060(380)$ \\
\hline Range & $\begin{array}{c}1,665-62,160 \\
(45-1,680)\end{array}$ & $\begin{array}{c}7,400-37,000 \\
(200-1,000)\end{array}$ & $\begin{array}{c}11,840-60,310 \\
(320-1,630)\end{array}$ & $\begin{array}{c}1,665-62,160 \\
(45-1,680)\end{array}$ \\
\hline \multicolumn{5}{|l|}{ Histology } \\
\hline Carcinoids & $482(44.1 \%)$ & $61(43.3 \%)$ & $145(48.2 \%)$ & $688(44.8 \%)$ \\
\hline pNET & $326(29.9 \%)$ & $26(18.4 \%)$ & $88(29.2 \%)$ & $440(28.7 \%)$ \\
\hline Rare NET & $100(9.1 \%)$ & $24(17.0 \%)$ & $26(8.6 \%)$ & $150(9.8 \%)$ \\
\hline Unknown primary & $185(16.9 \%)$ & $30(21.3 \%)$ & $42(14.0 \%)$ & $257(16.7 \%)$ \\
\hline Diabetes mellitus at baseline & $124(11.3 \%)$ & 24 (17.0\%) & $38(12.6 \%)$ & $186(12.1 \%)$ \\
\hline
\end{tabular}


TABLE 2

Predictors for Diabetes Mellitus $(n=1,535)$

\begin{tabular}{|c|c|c|}
\hline Variable & $\mathrm{HR}^{*}$ & $P$ \\
\hline Sex (female vs. male) & $1.102(0.767-1.795)$ & 0.698 \\
\hline Age (per y) & $0.998(0.978-1.017)$ & 0.806 \\
\hline Duration of disease (per mo) & $1.002(0.997-1.007)$ & 0.393 \\
\hline Previous surgery (vs. no surgery) & $0.973(0.579-1.633)$ & 0.916 \\
\hline Previous chemotherapy (vs. no chemotherapy) & $0.712(0.394-1.287)$ & 0.261 \\
\hline Previous radiation (vs. no radiation) & $0.679(0.251-1.840)$ & 0.447 \\
\hline${ }^{177}$ Lu-DOTATOC (vs. ${ }^{90} Y$-DOTATOC) & $0.000(0.000-0.498)$ & - \\
\hline Combination treatment (vs. ${ }^{90} \mathrm{Y}$-DOTATOC) & $1.023(0.542-1.931)$ & 0.945 \\
\hline Cumulative activity (per $1 \mathrm{mCi} / 37 \mathrm{MBq}$ ) & $1.000(0.999-1.001)$ & 0.622 \\
\hline pNET (vs. carcinoid) & $2.306(1.364-3.897)$ & 0.002 \\
\hline Rare NET (vs. carcinoid) & $0.796(0.261-2.431)$ & 0.688 \\
\hline Unknown primary (vs. carcinoid) & $0.586(0.222-1.550)$ & 0.282 \\
\hline \multicolumn{3}{|c|}{$\begin{array}{l}{ }^{\star} \text { Estimates for each variable have been adjusted for all other variables listed. } \\
\text { pNet = pancreatic neuroendocrine tumors. } \\
\text { Data in parentheses are } 95 \% \text { Cls. }\end{array}$} \\
\hline
\end{tabular}

patients, 72 patients $(5.3 \%)$ developed diabetes mellitus after DOTATOC treatment. Thereby, the only clinical predictor for developing diabetes mellitus was the diagnosis of pancreatic NET (Table 2).

The risk for developing diabetes mellitus was significantly higher before than after DOTATOC treatment (estimate, 0.0032, estimation of difference between a binomial- and the Aalen-
Johansen estimator, $P<0.001$; Fig. 3A). Sensitivity analyses including patients with or without pancreatic surgery confirmed these results.

Nine patients were lost to follow-up, 3 of whom had diabetes mellitus before DOTATOC treatment. A worst-case-scenario analysis with the remaining 6 patients assigned as diabetes mellitus cases generated results in line with the primary

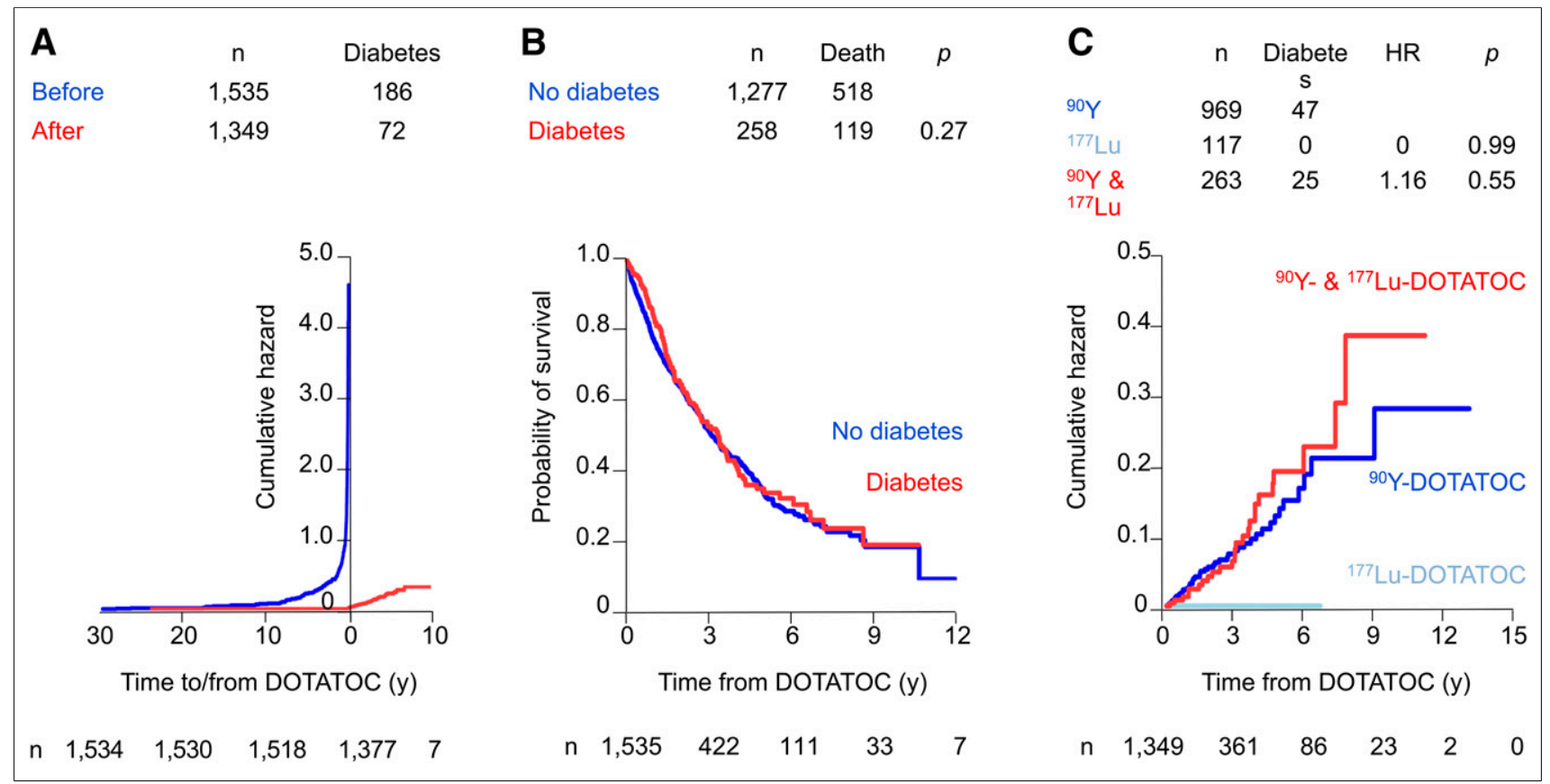

FIGURE 3. (A) Estimation of difference between binomial- and Aalen-Johansen estimator comparing incidence of diabetes mellitus before and after DOTATOC treatment showing significant peak before treatment and no significant increase after treatment. (B) Survival after DOTATOC in patients with and without diabetes mellitus showing no significant difference. (C) Cumulative hazard of diabetes mellitus after 3 treatment modalities showing cumulative hazard of 0 after ${ }^{177}$ Lu-DOTATOC and similar cumulative hazard after treatments with ${ }^{90} \mathrm{Y}-\mathrm{DOTATOC}$ and ${ }^{90} \mathrm{Y}$ plus ${ }^{177}$ Lu DOTATOC. 
analysis (HR, 1.09; 95\% CI, 0.58-2.06; proportional hazard regression, $n=1,093 ; P=0.78)$.

\section{Diabetes Mellitus and Mortality}

Overall, 637 of 1,535 patients $(41.5 \%)$ died, and $9(0.6 \%)$ patients were not available for follow-up. The median survival time after the start of DOTATOC treatment was 41.9 mo $(95 \%$ CI, 37.3-45.3 mo). Patients with and without diabetes mellitus had a similar survival, as confirmed by multivariate analyses (HR, 1.125; 95\% CI, 0.913-1.386; proportional hazard Cox regression model, $n=1,535 ; P=0.27$; Fig. $3 \mathrm{~B})$ and univariate analyses $(\log$ rank test, $n=1,535, P=0.50$ ).

\section{Diabetes Mellitus After ${ }^{177}$ Lu-DOTATOC or ${ }^{90}$ Y-DOTATOC}

During follow-up, diabetes mellitus developed in 47 of 969 patients $(4.9 \%)$ who received ${ }^{90}$ Y-DOTATOC and in 25 of 263 patients $(9.5 \%)$ who received ${ }^{90}$ Y-DOTATOC plus ${ }^{177} \mathrm{Lu}-$ DOTATOC. None of the 117 patients $(0 \%)$ who received ${ }^{177} \mathrm{Lu}$-DOTATOC developed diabetes mellitus (HR, 0.000; SE, 0.182; profile likelihood 95\% CI, 0.000-0.498; proportional hazard regression and profile likelihood, $n=117 ; P<$ 0.001; Fig. 3C).

After ${ }^{90}$ Y-DOTATOC, increased glucose levels (estimate, 0.038; linear mixed model, $n=70 ; P=0.004$; Supplemental Fig. 1A [supplemental materials are available at http://jnm.snmjournals.org]) were found but no significant changes in C-peptide levels (estimate, -0.032 ; linear mixed model, $n=70 ; P=0.485$; Supplemental Fig. 1B). After ${ }^{177}$ Lu-DOTATOC, no significant changes in glucose levels (estimate, 0.044; linear mixed model, $n=71 ; P=0.122$; Supplemental Fig. 1C) or C-peptide levels (estimate, 0.011; linear mixed model, $n=71 ; P=0.950$; Supplemental Fig. 1D) were found.

\section{DISCUSSION}

The present results revealed no increased risk of developing diabetes mellitus after somatostatin receptor-targeted radiopeptide therapy in NET. Conversely, the results suggest a higher risk of developing diabetes mellitus in patients before radiopeptide therapy. This observation may in part be due to a stringent diagnostic work-up coinciding with detection of NET or exposure to therapies with diabetogenic potential before radiopeptide treatment. Furthermore, there is no evidence of increased mortality in patients developing diabetes mellitus after radiopeptide therapy. Finally, the comparison of different treatment regimens suggests a lower diabetogenic potential of ${ }^{177} \mathrm{Lu}$ - over ${ }^{90} \mathrm{Y}-$ based therapies.

\section{Strengths and Limitations}

Strengths of the present study include the large patient number, the systematic data assessment, and the thorough follow-up, all of which contributed to robust statistical analyses. These analyses did not detect effects on diabetes mellitus incidence or diseasespecific mortality. However, these analyses do not exclude the possibility of such effects, which might be detectable by a much larger trial.

Further strengths include a comparison of ${ }^{90}$ Y-DOTATOC and ${ }^{177} \mathrm{Lu}$-DOTATOC, which used clinically relevant endpoints and biomarkers and which was supported by a worst-case-scenario analysis.

However, the present study was not a randomized trial and has the known limitations of a nonrandomized noncontrolled study.
Furthermore, the diagnosis of diabetes was obtained from the referring physicians. In patients in whom the presence of diabetes mellitus was uncertain, the referring physicians were contacted again to provide clarification. Systematic assessment of fasting plasma glucose and oral glucose tolerance tests were not feasible in stage IV cancer patients, who traveled to undergo oncologic treatment. Also, an ascertainment bias, because primary care physicians may have decreased their surveillance for diabetes mellitus in patients with the more serious neoplastic illness, cannot be excluded.

\section{Comparisons with Other Studies}

To the best of our knowledge, this is the first study to systematically assess the risk for developing diabetes mellitus after radiopeptide therapy and its disease-specific mortality. One report of 131 patients did not find changes in serum $\mathrm{HbA} 1_{c}$ after radiopeptide therapy (12), which is in line with our results.

\section{Implications}

In the clinical routine, diabetic patients should not be excluded from somatostatin receptor-targeted radiopeptide therapy. However, ${ }^{177} \mathrm{Lu}$-based treatment regimens might be considered in diabetic patients or those at high risk for developing diabetes, especially if other diabetogenic treatment modalities are part of their therapeutic regimen. On the other hand, increased awareness of glycemic control might be considered after ${ }^{90}$ Y-based treatment regimens in patients with prediabetic metabolism.

Further research that focuses on quality-of-life aspects related to glycemic control and on the recovery of diabetes mellitus and catabolic metabolism after completion of radiopeptide treatment might be valuable. Future studies should also implement data on comorbidities and medication with potential influence on diabetes mellitus.

Until additional studies are available, guidelines should consider somatostatin receptor-targeted radiopeptide therapy as relatively safe in regard to alterations of glucose metabolism. Routine assessment for diabetogenic toxicity after radiopeptide therapy in general does not seem justified based on the current results.

\section{CONCLUSION}

The current results suggest that receptor-targeted radiopeptide therapy is relatively safe in regard to alterations of glucose metabolism. Radiopeptide therapy does not seem to increase the risk of developing diabetes mellitus in NET patients, whereas the development of diabetes mellitus in NET patients after receptortargeted radiopeptide therapy does not seem to result in increased mortality.

\section{DISCLOSURE}

No potential conflict of interest relevant to this article was reported.

\section{ACKNOWLEDGMENTS}

We are grateful to our nursing, laboratory, and technical staff for their support in patient care, preparation of radiopharmaceuticals, 
and acquisition of scans. Furthermore, we thank Elisabeth OldaniSuter, Fausta Chiaverio, and Matthias Briel for their valuable contribution at the early stage of the project.

\section{REFERENCES}

1. Nicolas G, Giovacchini G, Muller-Brand J, Forrer F. Targeted radiotherapy with radiolabeled somatostatin analogs. Endocrinol Metab Clin North Am. 2011;40:187-204.

2. Imhof A, Brunner $\mathrm{P}$, Marincek $\mathrm{N}$, et al. Response, survival, and long-term toxicity after therapy with the radiolabeled somatostatin analogue $\left[{ }^{90} \mathrm{Y}-\right.$ DOTA]-TOC in metastasized neuroendocrine cancers. J Clin Oncol. 2011;29:2416-2423.

3. Villard L, Romer A, Marincek N, et al. Cohort study of somatostatin-based radiopeptide therapy with $\left[{ }^{90} \mathrm{Y}\right.$-DOTA]-TOC versus [ ${ }^{90} \mathrm{Y}$-DOTA]-TOC plus $\left[{ }^{177} \mathrm{Lu}-\mathrm{DOTA}\right]-\mathrm{TOC}$ in neuroendocrine cancers. J Clin Oncol. 2012;30:11001106.

4. Romer A, Seiler D, Marincek N, et al. Somatostatin-based radiopeptide therapy with [ $\left.{ }^{177} \mathrm{Lu}-\mathrm{DOTA}\right]-\mathrm{TOC}$ versus $\left[{ }^{90} \mathrm{Y}\right.$-DOTA]-TOC in neuroendocrine tumours. Eur J Nucl Med Mol Imaging. 2014;41:214-222.
5. Bernard V, Lombard-Bohas C, Taquet MC, et al. Efficacy of everolimus in patients with metastatic insulinoma and refractory hypoglycemia. Eur J Endocrinol. 2013;168:665-674.

6. Fiebrich HB, Siemerink EJ, Brouwers AH, et al. Everolimus induces rapid plasma glucose normalization in insulinoma patients by effects on tumor as well as normal tissues. Oncologist. 2011;16:783-787.

7. Barone BB, Yeh HC, Snyder CF, et al. Long-term all-cause mortality in cancer patients with preexisting diabetes mellitus: a systematic review and meta-analysis. JAMA. 2008;300:2754-2764.

8. Reubi JC, Waser B, Schaer JC, Laissue JA. Somatostatin receptor sst1-sst5 expression in normal and neoplastic human tissues using receptor autoradiography with subtype-selective ligands. Eur J Nucl Med. 2001;28:836846.

9. Andersen PK, Perme MP. Pseudo-observations in survival analysis. Stat Methods Med Res. 2010;19:71-99.

10. Terry M. Therneau PMG. Modeling Survival Data: Extending the Cox Model. 1st ed. New York, New York: Springer-Verlag; 2000:.

11. William S. Cleveland SJD, Eric Grosse. Regression by local fitting: methods, properties, and computational algorithms. J Econom. 1988;37:87-114.

12. Kwekkeboom DJ, Teunissen JJ, Bakker WH, et al. Radiolabeled somatostatin analog $\left[{ }^{177} \mathrm{Lu}\right.$-DOTA0,Tyr3] octreotate in patients with endocrine gastroenteropancreatic tumors. J Clin Oncol. 2005;23:2754-2762. 